EESTI NSV TEADUSTE AKADEEMIA TOIMETISED. XI KÖIDE FOOSIKALIS-MATEMAATILISTE JA TEHNILISTE TEADUSTE SEERIA, 1962, NR. $?$

ИЗВЕСТИЯ АКАДЕМИИ НАУК ЭСТОНСКОИ ССР. ТОМ ХІ СЕРИЯ ФИЗИКО-МАТЕМАТИЧЕСКИХ И ТЕХНИЧЕСКИХ НАУК, 1962, № 2

\title{
СИСТЕМА КРУГЛЫХ ТУРБУЛЕНТНЫХ СТРУЙ В СПУТНОМ ПОТОКЕ
}

\section{Х. Суи}

\section{Ю. В. ИВАНОВ,}

\section{доктор технических наук}

В статье приводятся результаты экспериментального изучения закономерностей развитня системы параллельных круглых турбулентных изотермических воздушных струй в спутном однородном потоке той же среды. Целью работы являлось установление влияння относительного шага между струями $s / d$ на закономерности развития струи в спутном потоке. Эта задача представляет определенный научный и практнческий интерес.

\section{Экспериментальная установка}

Схема экспериментальной установки приведена на фиг. 1. Ее основную часть составляет аэродинамическая труба диаметром $\mathrm{D}=700$ мм, создающая воздушный поток. В него помещена система спутных струй, выходящих из девяти круглых трубок постоянного диаметра (именуемых в дальнейшем соплами), укрепленных держателем параллельно в один ряд.

Использовалась такая сопловая система, в которой каждое сопло соединялось с распределительным коллектором при помощи отдельной резиновой трубки, начиная с держателей, находящихся вне потока. Это позволило регулировать скорость воздуха в трубках при помощи винтовых зажимов. Для того чтобы поток можно было считать бесконечным по отношению к системе сопел, диаметр последних был выбран небольшим - 8 мм. Сопла были разделены на две группы, выведенные по обе стороны аэродинамической трубы. Положение обеих групп сопел относительно друг друга фик. сировалось общим соединительным стержнем диаметром 10 мм. Длина рабочего участка, в котором изучался процесс перемешивания струй, равна 1100 мм.

Опыты проводилнсь с двумя различными скоростными полями при следующих отношениях скоростей струй и потока: $\lambda=v_{2} / v_{1}=2 ; 3 ; 5 ; 7 ; 10$ и $\infty$. Опыты с большими отношениями скоростей $(\lambda=7$ и 10$)$ проводились при средней скорости потока $v_{1}=4,5$, а с малыми значеннями $\lambda-$ при $v_{1}=7,4 \mathrm{~m} /$ сек. Скорость измерялась при помощи скоростной микротрубки. Скоростные поля потока имели указанные значения средней скорости в набегающем потоке перед системой струй. Сопловая группа, ока зывая сопротивление потоку, несколько снижала его скорость как в межсопловом пространстве, так частично и на некотором расстоянии по, потоку в области действия струй.

Для того чтобы развитие струй происходило в потоке, имеющем постоянную скорость, равную таковой для набегающего потока, нужно было бы увеличить скорость между отдельными соплами до средней скорости потока. Тогда при различных значениях относительных шагов между струями требовалось бы перед каждой группой сопел создавать поле особой формы. Поэтому при решении поставленной задачи был выбран вариант, в котором режим устанавливался по набегающей скорости 


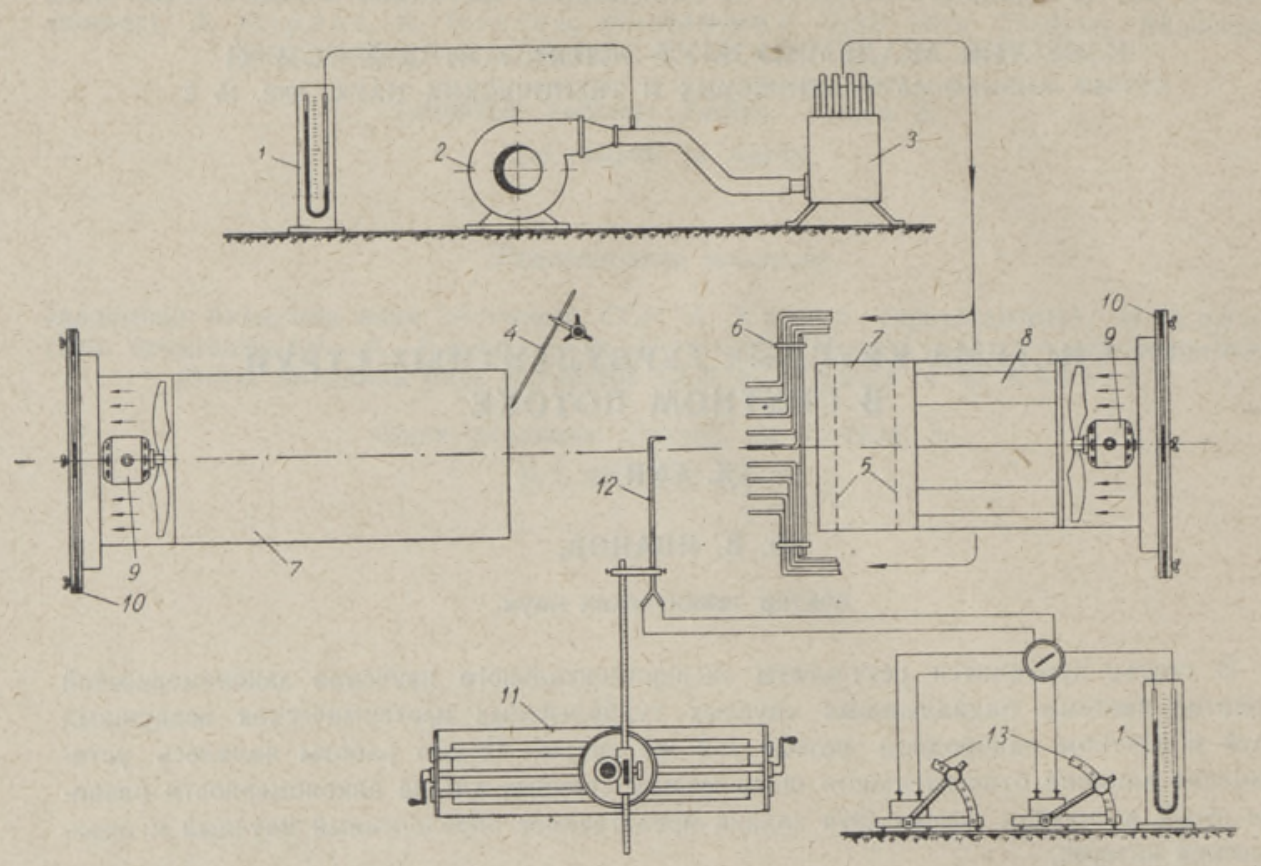

Фиг. 1. Схема экспериментальной установки и измерений: $1-U$-образный манометр; 2 - вентилятор среднего давления; 3 - распределительный коллектор; 4 - термометр для контроля температуры потока; 5 - выравнивающая решетка; 6 - сопловая система; 7 - аэродинамнческая труба; 8 - выравнивающее приспособление; 9 - аксиальный вентилятор; 10 - шибер для регулирования количества воздуха в основном потоке; 11 - координатник; 12 - микротрубка для измерения давления; 13 - микроманометр типа $\mathrm{MMH}$.

основного потока. При этом местные значения скоростей в межсопловом пространстве и вблизи сопел уменьшались не более чем на $12 \%$ от средней скорости основного потока. Изменения скоростей, имеющие место при больших значениях параметра $\lambda$, не столь суцественны, чтобы серьезно изменить зависимости, характерные для развития системы параллельных струй в спутном потоке при различных относительных шаrax.

Уменьшению влияния изменения скорости $v_{1}$ на исследуемый процесс способствовало то обстоятельство, что при уменьшении относительного шага, т. е. тогда, когда скорость потока сильнее всего снижается, струи сливались уже вблизи устья сопел.

В установке использовалось 9 сопел, но скорости нзмерялись только в средней струе как по оси, так и в различных ее сечениях до осей соседних струй, в двух гзаимно перпендикулярных плоскостях: по горизонтали $(z)$ и по вертикали $(y)$.

Коэффициент структуры струй $(a)$, исследуемых в работе, равнялся 0,09 .

\section{Результаты опытов}

Определяющим параметром развития отдельной изотермической струи является отношение скоростей $\lambda$. Развитие же системы струй зависит еще от одного определяющего параметра - относительного шага между струями. Понятно, что параллельные спутные струи влияют на изменение осевой скорости тем больше, чем меньше относительный шаг между ними.

В работе не ставилась цель точного определения значения относительного шага, при котором начинается влияние его на развитие струи. В ходе экспериментов выяснилось, что уже относительный шаг $s / d=8$ вызывает незначительный рост осе- 
вой скорости, да и то начиная примерно с расстояния $x / d=50$. Поэтому изучение влияния шага при значениях $s / d>8$ не производилось. Можно предполагать, что при дальнейшем увеличении относительного шага кривая затухания струи в системе доволінно скоро совпадет с кривой затухания отдельной струн.

Затухание единичной струи, развивающейся в спутном потоке, можно определить по формуле, приведенной в ранее опубликованной работе [1].

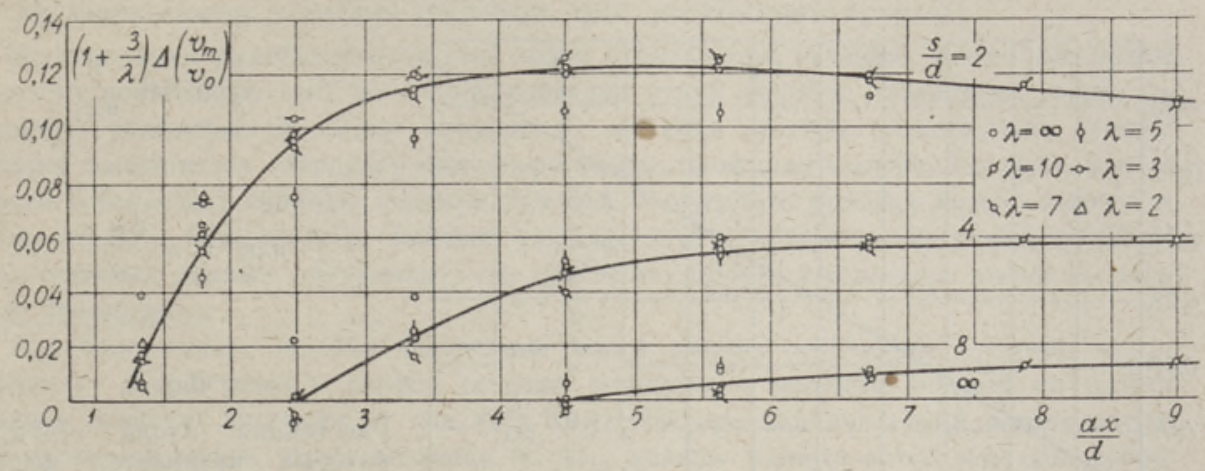

Фиг. 2. Влияние относительного шага между струями на осевую скорость струи.

На основе экспериментальных данных, полученных при различных относительных шагах, можно определить величину поправки для затухания осевой скорости $-\Delta\left(v_{m} / v_{0}\right)$. Под величиной поправки $\Delta\left(v_{m} / v_{0}\right)$ мы понимаем разность между кривыми затухания єсевых скоростей системы струй при различных относительных шагах и кривой затухания осевой скорости единичной струи $(s / d=\infty)$, полученными на всем участке длины струи в опытах с теми же значениями $\lambda$. Из этого условия вытекает, что при значеніи $s / d=\infty$ поправка равна нулю. Значения поправок построены по экспериментальным данным на фиг. 2. Из графика видно, что в принятых координатах экспернментальные точки опытов, при данном относительном шаге, располагаются таким обраsон, что через них оказалось возможным провести единую кривую, достаточно точно характеризующую величину поправки. Она зависит главным образом от относительного шага $s / d$ и в меньшей мере от отношения скоростей $\lambda$.

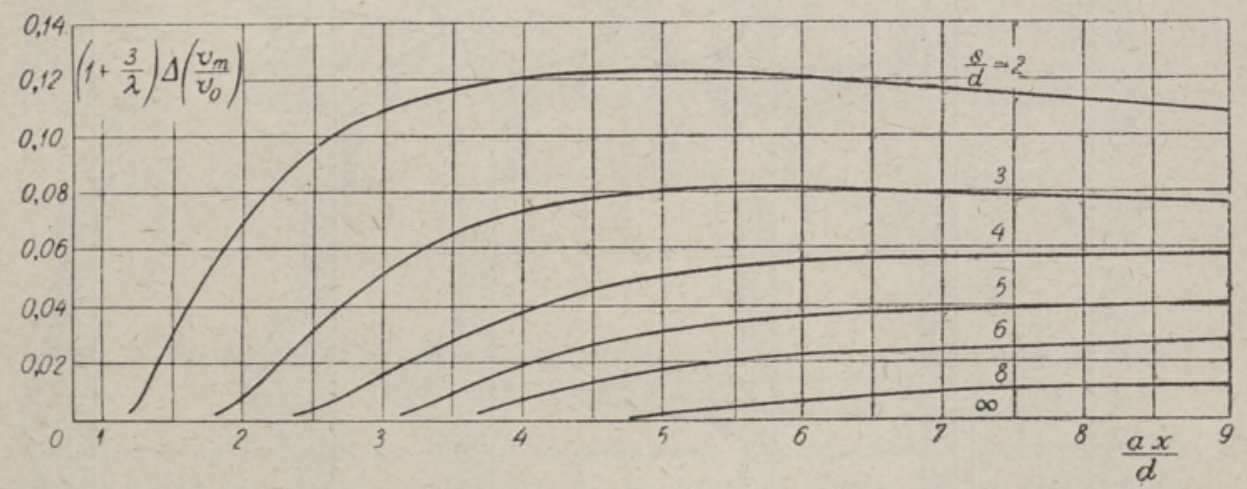

Фиг. 3. График для определения поправки $\Delta\left(v_{m} / v_{0}\right)$ в зависимости от относительного шага между струями.

Для определения этой поправки при различных относительных шагах $(s / d>2)$ она построена на фиг. 3 в виде сетки кривых для целых значений относительных шагов. 
Поправку для струй, размещенных с малым относительным шагом, нужно вводить уже вблизи устья. При $s / d=2 ; 4$ и 8 она должна вводиться соответственно с расстояния $a x / d \approx 1,2 ; 2,5$ и 4,6 или $x / d \approx 13 ; 28$ и 51 . Зависимость расстояний, измеренных вдоль оси струй, с которых начинается поправка на осевую скорость, от шага струи построена в координатах $1 / s, 1 / a x_{1}$ на фиг. 4. Қак видно из графика, это расстояние зависит от шага линейно:

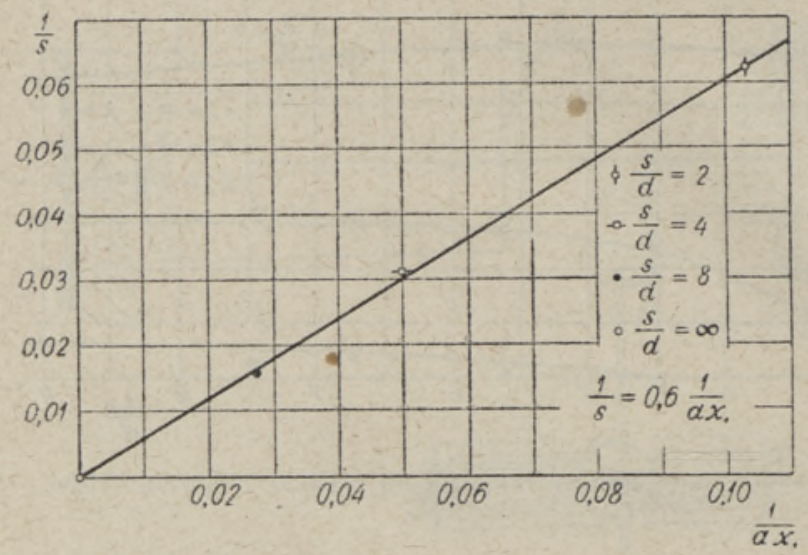

$$
\begin{aligned}
\frac{1}{s} & =0,6 \frac{1}{a x_{1}} \\
\text { или } x_{1} & =\frac{0,6 s}{a} .
\end{aligned}
$$

Фиг. 4.

Расстояння вдоль струн, с которых начннается влияние шага между струями на осевуяо скорость.

До этого расстояния величина поправки $\Delta\left(v_{m} / v_{0}\right)=0$. При больших относительных шагах величина поправки невелика, и при $s / d>8$ ее можно даже не учитывать.

Рассмотрим скоростные поля системы струй и их деформацию.

В зависимости от величины относительного шага скоростные поля отдельных струй на том или ином расстоянии от устья сливаются в общий поток. На фиг. 5 по-

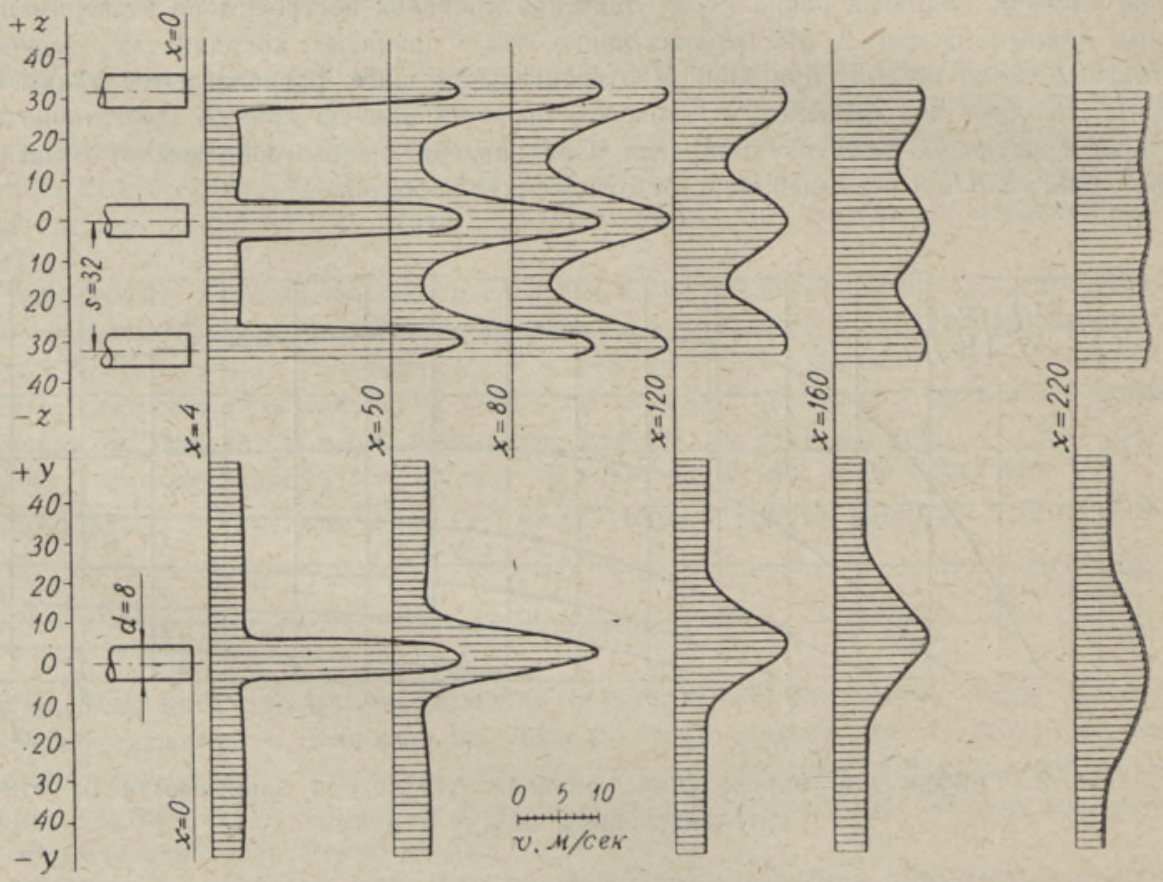

Фиг. 5. Скоростные поля системы круглых параллельных струй, развивающихся в спутном потоке при $s / d=4$ и $\lambda=7$. 
строены скоростные поля системы струй, замеренные при $s / d=4$ и $\lambda=7$ в горизонтальной и вертикальной плоскостях. Из графика видно, что струи, развивающиеся вблизи устья сопла, сохраняют свою индивидуальность. В -дальнейшем они начинают сливаться и на некотором расстоянии образуют единую сплошную струю. При уменьшении шага скоростные поля отдельных струй в горизонтальном сечении сливаются в общее поле уже вблизи устья сопла, в то время как в вертикальном сечении струя еще на значнтельном удалении сохраняет свой характерный профиль.

Исследования подобия скоростных полей в различных сечениях в горизонтальной плоскости показывают, что до соприкосновения струй подобие скоростных полей сохраняется полностью по всему сечению. С момента касания-межструйное сечение начинает заполняться сливающимися скоростными профилями струй. Скорость в этой области перерастает скорость основного потока. Постепенно разница между скоростью на оси струн и межструйном сечении уменьшается, пока совсем не исчезнет. Обработка опытных данных показывает, что при этом подобие скоростных полей на путн струи сохраняется.

Для определения подобия скоростных полей обычно используют величину $z_{\text {cp }}$ координату такой точки в данном сечении, избыточная скорость которой составляет половину значения скорости на оси $\left(0,5 \Delta v_{m}\right)$. Но при слиянии струй вскоре настунает такое положение, когда величина $0,5 \Delta v_{m}$ меньше минимального значения скорости между струями. В этом случае проводить такую обработку полей, замеренных в горизонтальном сечении, не представляется возможным.

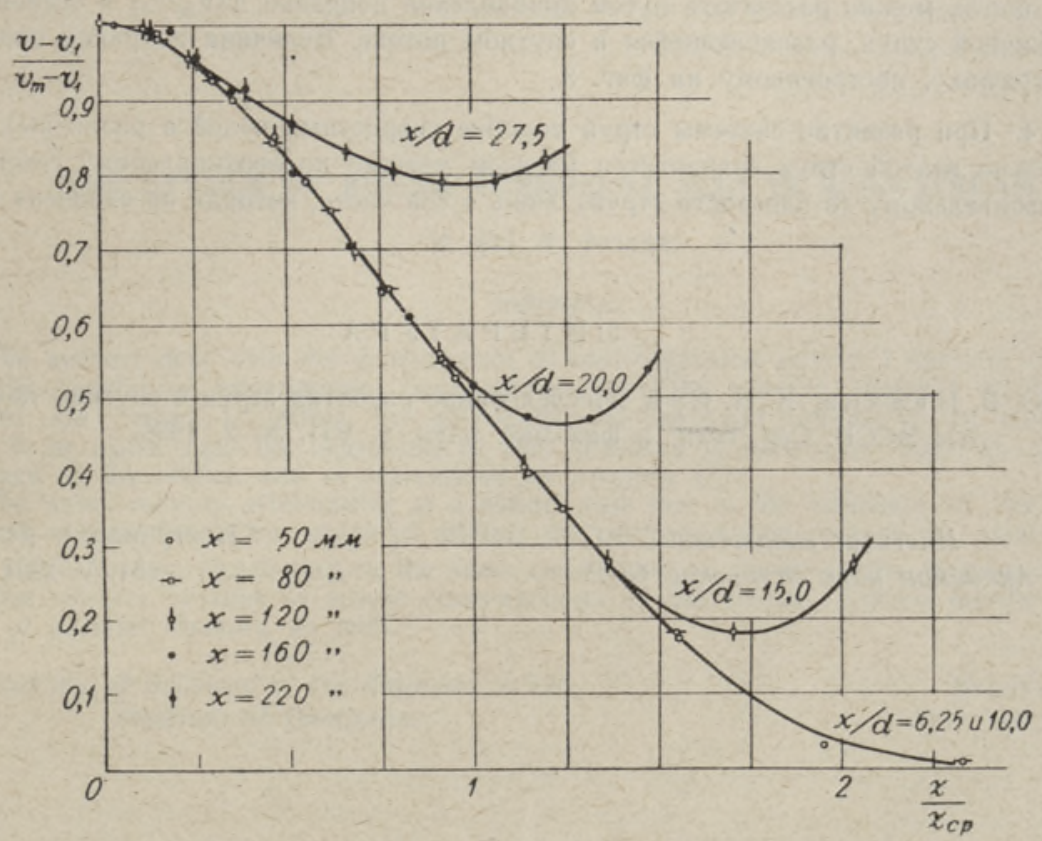

Фиг. 6. Профиль скоростных полей струи в различных горизонтальных сечениях при $s / d=4$ и $\lambda=7$.

Экспериментальные данные фиг. 5 использованы для построения универсального профиля подобия скоростных полей (фиг. 6), из которого видно, что по мере удаления от устья сопла не все сечения полностью укладываются на универсальную кривую. В сечения $x / d=6,25$ и 10,0 при $\lambda=7$ и $s / d=4$ подобие скоростных полей характеризуется полным совпадением опытных точек с теоретической кривой для единичной струи. При $x / d=15$ и 20 опытные точки занимают уже только верхнюю часть подобного нрофиля, а затем начинают отклоняться и загибаются вверх симметрично профилю соседней струи. 
При $x / d=27,5$ скорость в горизонтальном сечении (в плоскости параллельных струй) настолько выравнивается, что графическое определение $z_{\text {ср }}$ становится уже невозможным. Используя величину $z_{\text {ср }}$, найденную по измерениям в вертикальном сечении, удалось построить кривую подобного распределения скоростей и в этом сечении. Қак видно из графика, в этом случае можно говорить о подобии лишь в самой конечной части скоростного поля. В вертиқальном сечении подобие скоростных полей сохраняется полностью и при $x / d=27,5$, поэтому можно считать, что оно сохраняется на всем пути струн. Такие же результаты получены и при обработке остальных онытов.

\section{Выводы}

1. Затухание осевой скорости системы параллельных круглых струй, развивающихся в спутном потоке, зависит, кроме отношения скоростей $\lambda$, и от относительного шага между струями $s / d$. Система струй в потоке затухает медленнее единичной струи. С ростом относительного шага его влияние быстро уменьшается, и при значениях $s / d>8$ оно практически уже не сказывается.

2. Относительный шаг влияет на затухание осевой скорости системы струй, начиная с некоторого расстояния от устья сопла, которое пропорционально относительному шагу и определяется по уравнению (1).

3. Величину действительной скорости на оси струи при различных относительных шагах можно рассчитать путем прибавления поправки $\Delta\left(v_{m} / v_{0}\right)$ к осевой ско; эсти единичной струи, развивающейся в спутном потоке. Величина поправки определяется по графику, построенному на фиг. 3 .

4. При развитии системы струй подобие скоростных полей в различных сечениях отдельно взятой струи сохраняется на всем участке по вертикальному сечению, и по горизонтальному (в плоскости струй) лишь в той части, которая не охвачена слиянием.

\section{ЛИ Т Е Р А Т У Р А}

1. Ю. В. И в ан ов, Х. Н. С у й, Исследование развития струи в спутном потоке, Изв. АН ЭССР. Сер. техн. и физ.-мат. наук, т. VII, № 2, 1958.

Ннститут энергетики Академии наук Эстонской ССР
Поступила в редакцию 31. V 1961 


\title{
OMARATE TURBULENTSETE JUGADE SÜTEEM SAMASUUNALISES VOOLUSES
}

\author{
H. Sui \\ J. Ivanov, \\ tehniliste teaduste doktor \\ Resümee
}

Artiklis käsitletakse ümarate paralleelsete ōhujugade levimist ühtlases samasuunalises vooluses.

Katsetulemused näitavad, et jugadevaheline suhteline samm $s / d$ hakkab avaldama mōju joa telgkiiruse kustumisele tingimusel, et $s / d<8$, kusjuures selle mōju algus on märgatav alles teatud kaugusel düüsi suudmest ja määratakse valemiga (1).

Suhtelise sammu mōju tōttu aeglasemalt kustuvat telgkiirust on vōimalik leida samades tingimustes voolava üksiku joa telgkiirusele paranduse $\Delta\left(v_{m} / v_{0}\right)$ lisamise teel, mis on leitav graafikult joon. 3 . puhul.

Kiirusväljad joa üksikutes ristlōigetes jäävad sarnasteks ka paralleelsete jugade

\section{Eesti NSV Teaduste Akadeenia Energeetika Instituut}

Saabus toimetusse

31. V 1961

\section{THE SYSTEM OF ROUND TURBULENT JETS IN A CO-STREAM}

\author{
H. Sui, Y. Ivanov
}

\section{Summary}

The authors deal with the development of round parallel jets in a uniform co-stream.

The results of experiments have shown that the relative interval between the streams $s / d$ begins to affect the attenuation of the axial velocity in the condition if $s / d<8$, in which case the beginning of this influence is noticeable only at a certain distance of the orifice and is determined by formula (1).

The axial velocity attenuating at a slower rate due to the influence of the relative interval, can be found by means of adding the correction $\Delta\left(v_{m} / v_{0}\right)$ to the axial velocity of a single stream developing in the same conditions as shown in the diagram in fig. 3 .

The velocity profiles in single cross-sections of the stream remain similar in the case of parallel streams, as well.

Academy of Sciences of the Estonian S.S.R., Institute of Energetics

Received May 31 st, 1961 